\title{
artigo
}

\section{Fatores envolvidos na prática de renovação automática de receitas médicas no contexto da atenção básica}

\author{
Factors involved in the practice of automatic renewal of medical prescriptions in the context of primary care
}

Factores que intervienen en la práctica de la renovación automática de prescripciones médicas en el contexto de la atención primaria

\section{RESUMO}

Objetivo: Caracterizar os principais fatores envolvidos na prática de renovação automática de receitas médicas nas unidades de saúde da família no contexto da atenção básica. Métodos: Trata-se de uma revisão bibliográfica narrativa, utilizando-se de levantamento bibliográfico realizado através de consulta de bancos de dados como Google Scholar, PubMed e SciELO, com a estratégia: "Uso Indevido de Medicamentos", "Prescrição Inadequada", "Atenção Primária à Saúde", "Receitas Médicas de Controle Especial" e "Medicamentos sob Prescrição", utilizando-se "AND" como operador booleano. Também foram consultados pareceres dos Conselhos Regionais de Medicina, bem como a legislação vigente em torno da prescrição médica e renovação de receitas. Conclusão: Com isso, observa-se que a prática de renovação automática de receitas médicas deve ser combatida. Isto pode proporcionar efeitos colaterais importantes, pior controle das comorbidades e falsa sensação de cuidado, além de inúmeras implicações ético-legais.

DESCRITORES: Uso Indevido de Medicamentos; Prescrição Inadequada; Atenção Primária à Saúde; Receitas Médicas de Controle Especial; Medicamentos sob Prescrição.

\section{ABSTRACT}

Objective: To characterize the main factors involved in the practice of automatic renewal of medical prescriptions in family health units in the context of primary care. Methods: This is a narrative bibliographic review, using a bibliographic survey carried out by consulting databases such as Google Scholar, PubMed and SciELO "Misuse of Medicines", "Inappropriate Prescription", " Primary Health Care "', Special Control Medical Prescriptions "and" Prescription Drugs ", using" AND "as a Boolean operator. Opinions of the Regional Councils of Medicine were also consulted, as well as the legislation in force around medical prescription and prescription renewal. Conclusion: With this, it is observed that the practice of automatic renewal of medical prescriptions must be combated. This can provide important side effects, worse control of comorbidities and a false sense of care, in addition to numerous ethical and legal implications.

DESCRIPTORS: Misuse of Medicines; Inadequate prescription; Primary Health Care; Special Control Medical Prescriptions; Prescription Drugs.

\section{RESUMEN}

Objetivo: Caracterizar los principales factores que intervienen en la práctica de la renovación automática de prescripciones médicas en las unidades de salud de la familia en el contexto de la atención primaria. Métodos: Se trata de una revisión bibliográfica narrativa, mediante un relevamiento bibliográfico realizado mediante consulta de bases de datos como Google Scholar, PubMed y SciELO "Mal uso de medicamentos", "Prescripción inadecuada", 'Atención primaria de salud "', Recetas médicas de control especial "y" Medicamentos recetados ", utilizando" Y "como operador booleano. También se consultaron las opiniones de los Consejos Regionales de Medicina, así como la legislación vigente en materia de prescripción médica y renovación de prescripciones. Conclusión: Con esto, se observa que se debe combatir la práctica de renovación automática de prescripciones médicas. Esto puede proporcionar importantes efectos secundarios, un peor control de las comorbilidades y una falsa sensación de cuidado, además de numerosas implicaciones éticas y legales.

DESCRIPTORES: Uso indebido de medicamentos; Prescripción inadecuada; Primeros auxilios; Recetas médicas de control especial; Medicamentos con receta.

RECEBIDO EM: 09/12/2020 APROVADO EM: 21/12/2020 


\section{Luis Henrique Alves Gomes}

Graduando em Medicina, Centro Universitário CESMAC.

ORCID: 0000-0002-2540-9625

\section{Laisy Amorim Farias de Almeida}

Graduando em Medicina, Centro Universitário CESMAC.

ORCID: 0000-0002-2159-8895

\section{Caio Cézar Tenório Alves da Silva}

Graduando em Medicina, Centro Universitário UNIT.

ORCID: 0000-0002-3423-605X

\section{Alfredo Aurélio Marinho Rosa}

Médico. Graduação em Medicina. Universidade Federal de Alagoas, UFAL, Brasil. Centro Universitário Tiradentes, UNIT, Brasil. ORCID: 0000-0002-7938-1197

Alfredo Aurélio Marinho Rosa Filho

Médico. Graduação em Medicina. Centro Universitário Tiradentes, UNIT, Brasil. ORCID: 0000-0001-8060-221X

\section{INTRODUÇÃO}

$\mathbf{N}$ os tratamentos modernos, principalmente no contexto de mudanças demográficas e epidemiológicas, o uso correto dos medicamentos é essencial. No início do século 20, a transição das doenças agudas para as crônicas e o envelhecimento da população também estavam relacionados ao aumento do uso de medicamentos, principalmente ao uso contínuo de medicamentos ${ }^{1}$. No entanto, a mesma pessoa está usando cada vez mais vários medicamentos, o que leva a um aumento na incidência de interações medicamentosas e aumenta o risco de uso indevido $^{5}$. Esse contexto costuma gerar uma demanda por receitas na rede básica, que nem sempre consegue se organizar de forma adequada para reavaliar corretamente prescrições por vezes múltiplas, fornecidas a usuários comumente idosos e portadores de comorbidades ${ }^{7}$.

Nas unidades de saúde, as atualizações de prescrição são frequentemente realizadas sem avaliação clínica ${ }^{3}$. A existência de rotinas atualizadas inseridas no cotidiano dos serviços de atenção básica permite questionar os benefícios dessa prática para as pessoas no modelo atual ${ }^{1}$. A simples repetição das prescrições não atende ao objetivo principal da atualização: verificar o uso correto do medicamento, avaliar o efeito do tratamento e a dependência do medicamento, os efeitos colaterais são consideráveis, o risco para o paciente e a adesão ao tratamento são mínimos. Como na atenção básica, esse comportamento de renovação não só tem impacto logístico no processo de trabalho, mas também na própria saúde do paciente ${ }^{2 ; 10}$.

Mesmo em sistemas de saúde mais antigos e/ou com mais recursos, discute-se muito sobre o papel dos diferentes membros da equipe de saúde na atualização das prescrições e seus resultados na assistência integral ao paciente ${ }^{4}$. No entanto, esse assunto raramente é debatido no Brasil, e os autores descobriram que não há pesquisas sobre esse assunto em periódi$\cos$ brasileiros ${ }^{6 ; 11}$. Assim, percebe-se a pouca atenção dada a essa questão, talvez justificada por um fenômeno cultural que banaliza o uso de medicamentos e negligencia os seus riscos.

Os medicamentos combinados com fatores sociais (como nutrição, moradia e outros fatores) são muito importantes na modificação dos indicadores de saúde, pois estão relacionados à qualidade de vida de um indivíduo. É fundamental garantir sua continuidade no tratamento das doenças crônicas, mas também é fundamental reavaliar regularmente o quadro clínico, principalmente no tratamento medicamentoso prolongado ${ }^{8}$.

\section{OBJETIVO}

O presente estudo busca caracterizar os principais fatores envolvidos na prática de renovação automática de receitas médicas no contexto da atenção básica.

\section{MÉTODOS}

Trata-se de uma revisão da literatura, do tipo narrativa. As revisões narrativas podem levar em conta o campo teórico da produção do conhecimento, descrever e discutir os objetos de pesquisa. Desta forma, ao explicar e analisar pessoalmente criticamente o autor, analisa-se a literatura científica. Quais os aspectos ético-legais e sociais relacionados à renovação automática de receitas? Quais são as implicações da renovação automática de receitas no seguimento clínico desses pacientes? Estes foram os marcos teóricos deste estudo.

O material textual utilizado foi selecionado por meio de buscas parametrizadas, elegendo-se bases de dados de acesso livre como Scientific Electronic Library Online (SciELO), Literatura Latino-americana e do Caribe em Ciências da Saúde (Lilacs) e Google Scholar. O período da coleta de dados se deu entre novembro e dezembro de 2020. Aplicaram-se os descritores em ciências da saúde (DeCS) "Uso Indevido de Medicamentos", "Prescrição Inadequada", "Atenção Primária à Saúde", "Receitas 


\section{artigo}

Médicas de Controle Especial" e "Medicamentos sob Prescrição" utilizando-se "AND" como operador booleano.

A elegibilidade do material se deu por meio dos critérios de inclusão: textos completos do tipo artigos, sem recorte temporal e idioma português; e critérios de exclusão: estudos duplicados e estudos que não abordassem a temática. A seleção se deu com aplicação dos critérios de elegibilidade e, sequencialmente, leitura de títulos, resumos e, quando elegíveis, leitura completa dos artigos. A análise e interpretação dos artigos selecionados se deu pela sumarização dos achados e pela técnica da categorização temática, possibilitando a apresentação dos resultados.

\section{RESULTADOS}

Foram localizados 41 artigos a partir das buscas nas bases de dados. Após leitura dos títulos e resumos, restaram 15 artigos, conforme fluxograma dos resultados, representado na Figura 1 e Quadro de resultados. Posteriormente após leitura na íntegra nenhum estudo com relação específica com o tema foi localizado, entretanto, a partir da análise da literatura cinza quatro artigos foram incluídos na presente revisão.

$\mathrm{Na}$ análise dos artigos selecionados para revisão, destacou-se a escassa quantidade de artigos construídos no Brasil acerca da temática ao longo da década.

Figura 1: Fluxograma de busca dos artigos

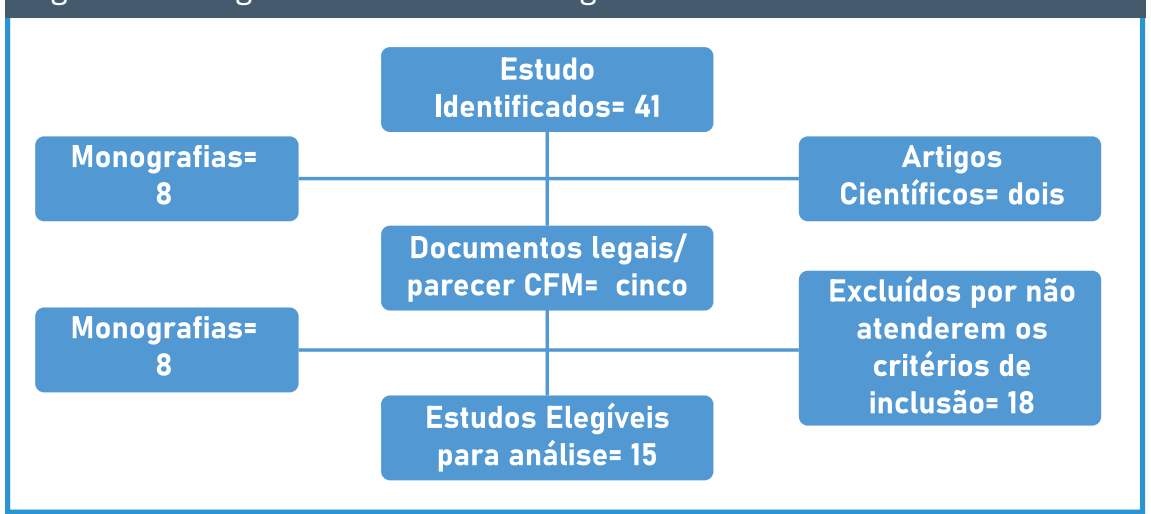

Fonte: Dados da pesquisa, 2020

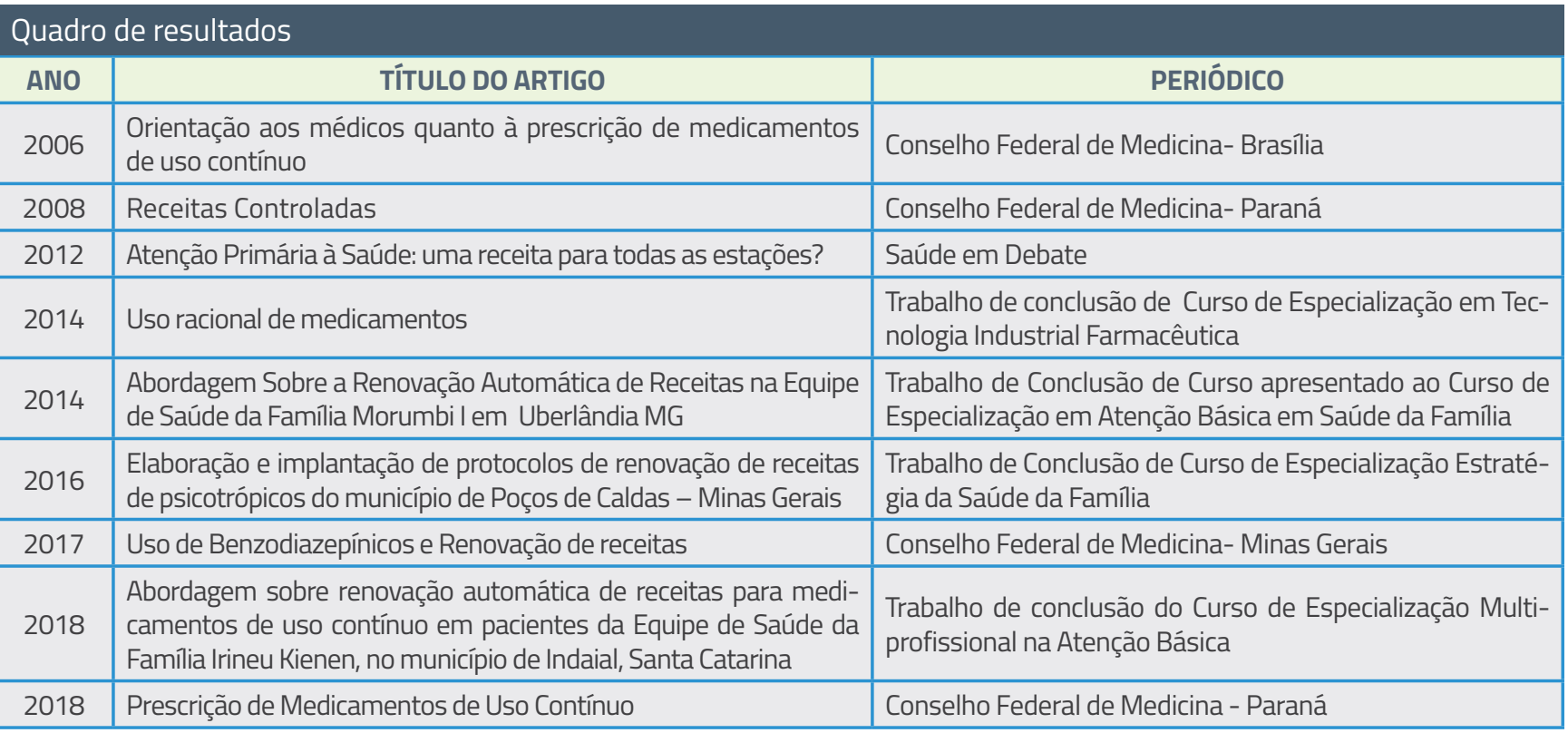




\begin{tabular}{|c|l|l|}
\hline 2018 & Repetição de receitas de psicofármacos e controladas & Conselho Federal de Medicina- Brasilia \\
\hline 2018 & Refil de prescrição na atenção primária à saúde: uma análise crítica. & Revista Médica de Minas Gerais \\
\hline 2018 & $\begin{array}{l}\text { Psicotrópicos e Ansiolíticos na Estratégia Saúde da Família (ESF) } \\
\text { Vila Nova }\end{array}$ & $\begin{array}{l}\text { Trabalho de Conclusão do Curso de Especialização Multi- } \\
\text { profissional na Atenção Básica }\end{array}$ \\
\hline 2018 & $\begin{array}{l}\text { Abordagem sobre renovação automática de receitas para medi- } \\
\text { camentos de uso contíno em pacientes da Equipe de Saúde da } \\
\text { Familia Irineu Kienen, no município de Indaial, Santa Catarina }\end{array}$ & $\begin{array}{l}\text { Trabalho de Conclusão do Curso de Especialização Multi- } \\
\text { profissional na Atenção Básica }\end{array}$ \\
\hline 2019 & $\begin{array}{l}\text { Projeto de Intervenção para Trabalhar com os Usuários a não Re- } \\
\text { novação Automática de Receitas pela Equipe de Saúde da Familia } \\
\text { Orlando Gangini em Planura- Minas Gerais }\end{array}$ & $\begin{array}{l}\text { Trabalho de Conclusão de Curso apresentado ao Curso de } \\
\text { Especializaçãa Gestão do Cuidado em Saúde da Familia }\end{array}$ \\
\hline 2019 & $\begin{array}{l}\text { Abuso de Medicamentos Psiquiátricos: Plano de Ação na Equipe } \\
\text { de Saúde da Familia Sevilha Bll, Ribeirão das Neves, Minas Gerais }\end{array}$ & $\begin{array}{l}\text { Trabalho de Conclusão de Curso apresentado ao Curso de } \\
\text { Especialização em estratégia em Saúde da Familia }\end{array}$ \\
\hline Fonte: Dados da Pesquisa, 2020. & \\
\hline
\end{tabular}

\section{DISCUSSÃO}

\section{Visão operacional da prescrição médica}

Existem inúmeros fatores socioculturais associados à renovação das prescrições médicas que vão de encontro aos normativos do Conselho Federal de Medicina (CFM) e consequentemente a boa prática profissional, através dos direitos e deveres da ética médica. A renovação de receitas médicas de pacientes com doenças crônicas depende de assistência próxima de qualidade e revisão terapêutica constante. Entretanto, observa-se um ciclo de renovação de prescrições anteriores, mesmo sem realização de consultas médicas, cuja finalidade seria de reavaliar e verificar se a indicação permanece válida. A prática de nova prescrição de medicamentos sem o devido cuidado e atenção médica dá-se o nome de Renovação Automática de Receitas Médicas ${ }^{4 ; 9 ; 10}$.

Embora muitos pacientes possam apresentar estabilidade no tratamento contínuo de suas comorbidades, a falta de reavaliação periódica se mostra inadequada e antagônica à boa prática médica. O uso de uma terapêutica contínua, deve ser acompanhada de perto pelo profissional médico, sendo avaliado também a resposta ofertada pela medicação, seja ela ineficaz ou não, o risco de efeitos colaterais e de interações medicamentosas, facilitado pelo não acompanhamento destes pacientes, além de resultar em menor adesão terapêutica, pois a cada encontro com profissional de saúde é uma oportu- nidade para esclarecimentos sobre condição de base e dúvidas sobre morbidade e prognóstico. A ausência deste cuidado é danosa ao paciente ${ }^{5 ; 6 ; 8}$.

Em função do sistema público apresentar volume laboral desproporcional ao contingente de profissionais médicos no sistema público de saúde, além de trabalhar a partir do cumprimente quantitativas e não qualitativas, o trabalho destes profissionais passa a se preocupar com volume, muitas vezes com cobertura de extensas comunidades com diversos problemas sociais, culturais, financeiros e biológicos. Desse modo, ocorre uma demanda reprimida, por inúmeros fatores, em que a quantidade de consultas é maior do que a mão de obra fornecida. Observa-se com isso, uma luta por fichas de consultas e um relaxamento quanto às condutas de prescrições daqueles pacientes que possuem uma comorbidade crônica ${ }^{12 ; 15}$.

Importante destacar ainda a visão operacional e legal das receitas médicas. Verificou-se ainda que o preenchimento e a prescrição médica em si obedecem a normas sanitárias que orientam validade das receitas de 30 dias a contar do dia de seu preenchimento (Portaria Anvisa $\mathrm{n}^{\circ}$ 6/1999, em seu artigo 85, parágrafo $1^{\circ}$, alínea "A"), colocando um limite temporal para a receita ${ }^{1 ; 2 ; 4 ; 9}$.

Quanto à quantidade, esta varia de acordo com o tipo de receituário exigido. Aqueles medicamentos sujeitos a controle por receituário comum carbonado $(\mathrm{C} 1-$ outras substâncias sujeitas a controle especial) têm suas receitas válidas por 30 dias a contar da data de seu preenchimento e quantidade máxima para 60 dias de tratamento; do mesmo modo aquelas do grupo "B" (B1, B2 - psicotrópicos e anorexígeno), com validade de 30 dias e quantidade máxima para 60 dias de tratamento (artigo 42 e parágrafos 44 a 49, e parágrafos da Portaria Anvisa no 344/1998) $)^{1 ; 9}$.

A prescrição médica deve ser sempre precedida de uma avaliação clínica, e para uma boa prática médica, não se deve receitar nenhum tipo de medicamento sem ver o paciente. O que deve ser feito para os pacientes com doenças que requeiram uso contínuo de psicofármacos de quaisquer naturezas é a prescrição para um máximo de 90 dias, com liberação controlada a cada 30 dias, ficando os pacientes na obrigação de voltar para nova consulta e nova prescrição a cada três meses, a ausência de um fluxograma para atendimento de pacientes com doenças crônicas, leva a esse descumprimento ${ }^{1 ; 3 ; 4}$.

A falta de flexibilidade no horário de atendimento da Unidade básica de saúde, faz com que a classe assalariada tenha dificuldades para se apresentar nos horários estabelecidos, o que só aumenta esse desfecho. No entanto, muitos dos pacientes não fazem a visita à unidade de forma adequada devido ao costume e a comodidade de em tempos passados terem a receita renovada automaticamente ${ }^{78 ; 11}$.

As prescrições para 30 ou 60 dias, conforme preconiza a Portaria Anvisa ${ }^{\circ}$ 


\section{artigo}

Gomes, L.H.A.; Almeida, L.A.F.; Alves da Silva, C.C.T.; Rosa, A.A.M.; Filho-Rosa, A.A.M.;

Fatores envolvidos na prática de renovação automática de receitas médicas no contexto da atenção básica

344/1998, continuarão valendo, porque as reconsultas de controle dependem de muitas variáveis e tem caráter prognóstico, devendo ficar a critério do médico estabelecer o tempo de retorno. Quando optar por 90 dias, deve ficar consignado na receita "VÁLIDA PARA 90 DIAS", fazendo o mesmo assentamento no prontuário $^{1 ; 4 ; 9}$.

A falta de conhecimento da equipe e dos médicos da necessidade de reavaliação periódica, juntamente com a carência de atividades para educação em saúde sobre a temática, faz com que a situação seja negli- genciada e práticas como essas sejam continuadas, o que traz consequências éticas e sociais, além de possíveis danos irreversíveis ao paciente $\mathrm{e}^{2 ; 3 ; 10 ; 14}$.

\section{CONCLUSÃO}

Com isso, observa-se que a prática de renovação automática de receitas médicas deve ser combatida, pois está associada a ausência de reavaliação periódica e programada dos pacientes. Isto pode proporcionar efeitos colaterais importantes, pior controle das comorbidades e falsa sensa- ção de cuidado, além de inúmeras implicações ético-legais. A prescrição médica faz parte do ato médico e não há ato médico sem consulta e deve se reforçar isso diariamente. Identificou-se como fatores propagadores deste ciclo os comportamentos do paciente, os hábitos culturais, pressão sobre a equipe e o processo de trabalho da instituição de saúde. Somente com educação continuada e conscientização da situação-problema será possível combater a renovação automática de receitas e oferecer uma nova perspectiva com segurança e saúde de qualidade aos pacientes.

\section{REFERÊNCIAS}

1. Da Rocha R Ana Leda. Uso racional de medicamentos. Rio de Janeiro: Instituto de Tecnologia em Fármacos, 2014. Trabalho de conclusão de Curso de Especialização em Tecnologia Industrial Farmacêutica.

2. Brito FN Rosana. Abordagem sobre renovação automática de receitas para medicamentos de uso contínuo em pacientes da Equipe de Saúde da Família Irineu Kienen, no município de Indaial, Santa Catarina. Florianópolis: Universidade Federal De Santa Catarina, 2018. Trabalho de conclusão do Curso de Especialização Multiprofissional na Atenção Básica.

3. Cruz BAB. Elaboração e implantação de protocolos de renovação de receitas de psicotrópicos do município de Poços de Caldas - Minas Gerais. Belo Horizonte: Universidade Federal de Minas Gerais, 2016. Trabalho de Conclusão de Curso de Especialização Estratégia da Saúde da Família.

4. Prescrição de Medicamentos de Uso Contínuo. PARECER Nº 2643/2018. Conselho Federal de Medicina - Paraná. De fevereiro de 2018. Disponível em: https://sistemas.cfm.org.br/normas/arquivos/pareceres/PR/2018/2643_2018.pdf

5. Receitas Controladas. PARECER No 2017/2008. Conselho Federal de Medicina- Paraná. De novembro de 2008. Disponível em: https://sistemas.cfm.org.br/normas/visualizar/pareceres/ PR/2008/2017

6. Orientação aos médicos quanto à prescrição de medicamentos de uso contínuo. PARECER No 12/06. Conselho Federal de Medicina- Brasília. De agosto de 2006. Disponível em: https://sistemas.cfm.org.br/normas/arquivos/pareceres/ BR/2006/12_2006.pdf

7. Repetição de receitas de psicofármacos e controladas. PARECER No 20/2018. Conselho Federal de Medicina- Brasília. De maio De 2018. Disponivel em: https://sistemas.cfm.org.br/normas/arquivos/pareceres/BR/2018/20_2018.pdf

8. Uso de Benzodiazepínicos e Renovação de receitas. PARECER No 126/2017. Conselho Federal de Medicina- Minas Gerais. De julho de 2017. Disponivel em: https:/sistemas.cfm.org.br/normas/arquivos/pareceres/MG/2017/126_2017.pdf
9. Reis I, Alves L, Da Cunha L, Cavalli, De Aguiar Raphael. Refil de prescrição na atenção primária à saúde: uma análise crítica. Revista Médica de Minas Gerais [revista em Internet]. 7 de março de 2018. Acesso em 20 de dezembro de 2020; 28. Disponível em: http://rmmg.org/artigo/detalhes/2360

10. Pelaez Y. Psicotrópicos e Ansiolíticos na Estratégia Saúde da Família (ESF) Vila Nova. Florianópolis: Universidade Federal de Santa Catarina, 2018. Trabalho de Conclusão do Curso de Especialização Multiprofissional na Atenção Básica.

11. Perez R. Projeto de Intervenção para Trabalhar com os Usuários a não Renovação Automática de Receitas pela Equipe de Saúde da Família Orlando Gangini em Planura- Minas Gerais. Minas Gerais: Universidade Federal do Triângulo Mineiro, 2019. Trabalho de Conclusão de Curso apresentado ao Curso de Especialização Gestão do Cuidado em Saúde da Família.

12. Brito R. Abordagem sobre renovação automática de receitas para medicamentos de uso contínuo em pacientes da Equipe de Saúde da Família Irineu Kienen, no município de Indaial, Santa Catarina. Florianópolis: Universidade Federal de Santa Catarina, 2018. Trabalho de Conclusão do Curso de Especialização Multiprofissional na Atenção Básica.

13. De Carvalho, T. Abordagem Sobre a Renovação Automática de Receitas na Equipe de Saúde da Família Morumbi I em Uberlândia MG. Minas Gerais: Universidade Federal do Triângulo Mineiro, 2014. Trabalho de Conclusão de Curso apresentado ao Curso de Especialização em Atenção Básica em Saúde da Família.

14. Mesquita L. Abuso de Medicamentos Psiquiátricos: Plano de Ação na Equipe de Saúde da Família Sevilha BII, Ribeirão das Neves, Minas Gerais. Minas Gerais: Universidade Federal de Minas Gerais, 2019. Trabalho de Conclusão de Curso apresentado ao Curso de Especialização em estratégia em Saúde da Família.

15. Paim J. Atenção Primária à Saúde: uma receita para todas as estações?. Saúde em Debate [revista em Internet]. Setembro de 2012. Acesso em: 19 de novembro de 2020; 36. Disponível em: https://www.scielosp.org/pdf/sdeb/2012.v36n94/343-347/pt 